\title{
Antitumor effect of microbubbles enhanced by low frequency ultrasound cavitation on prostate carcinoma xenografts in nude mice
}

\author{
YU WANG, BING HU, XUEHONG DIAO and JIZHEN ZHANG \\ Department of Ultrasound in Medicine, Shanghai Jiaotong University Affiliated Sixth People's Hospital, \\ Shanghai 200233, P.R. China
}

Received September 8, 2011; Accepted October 21, 2011

DOI: $10.3892 /$ etm.2011.377

\begin{abstract}
The aim of this study was to investigate the antitumor effect induced by low frequency $(20 \mathrm{kHz})$ ultrasound (US) radiation combined with intravenous injection of microbubbles (Mbs) on prostate carcinoma Du145 xenografts in nude mice. Du145 prostate tumors were percutaneously implanted in 40 nude mice, which were randomly divided into 4 groups ( $\mathrm{n}=10$ each): US+Mbs, US, Mbs and control groups. The mice in the US+Mbs group were treated with $20 \mathrm{kHz}$, $200 \mathrm{~mW} / \mathrm{cm}^{2}$ US radiation and with $0.2 \mathrm{ml} \mathrm{Mbs} \mathrm{injected} \mathrm{intra-}$ venously. Mice in the US and Mbs groups were only treated with US radiation and injection of Mbs, respectively. Tumors were measured with sonography, and the ratio of antitumor growth was calculated. The mice were sacrificed 14 days after treatment. Specimens of the tumor tissues were observed pathologically using light microscopy and transmission electron microscopy. Microvessel density and the average optical density of vascular endothelial growth factor were compared among groups by immunohistochemistry. The average gross tumor volume of the US+Mbs group was significantly reduced compared with the other groups following treatment $(\mathrm{P}<0.05)$. The ratio of the antitumor growth in the US+Mbs group was significantly greater than that of the US and Mbs group $(\mathrm{P}<0.05)$. Histological examination showed signs of tumor cell injury in the US+Mbs group. Examination by electron microscopy revealed vessel injury in the endothelium in the tumors treated with US+Mbs. Microvessel density and the average optical density of vascular endothelial growth factor in the US+Mbs group were significantly less than that of other groups $(\mathrm{P}<0.05)$. In conclusion, low frequency US of $20 \mathrm{kHz}$ radiation combined with $\mathrm{Mbs}$ may be used to inhibit the
\end{abstract}

Correspondence to: Dr. Bing Hu, Department of Ultrasound in Medicine, Shanghai Jiaotong University Affiliated Sixth People's Hospital, 600 Yi Shan Road, Shanghai 200233, P.R. China

E-mail: binghuzz@gmail.com

Key words: low frequency ultrasound, microbubbles, cavitation, prostate carcinoma growth of human prostate carcinoma xenografts in nude mice, and the effect is likely realized through microvessel destruction caused by cavitation.

\section{Introduction}

High-intensity focused ultrasound (HIFU) surgery was first used to created focal lesions deep in liver tissue and was further developed by a group headed by William Fly in Illinois in the 1950s (1). At the point where the ultrasound (US) waves are focused, sudden and intense absorption of the US beam creates a rapid elevation in temperature, which destroys the cells located at the targeted area without damaging tissue elsewhere in the path of the beam. HIFU has been used to treat glaucoma in human patients (2) and for ablation of prostatic tissue in dogs (3). Clinical studies also explored the use of HIFU for the transrectal treatment of benign prostatic hyperplasia and prostate cancer (4-6). The main disadvantage of HIFU is that only a small amount of tissue is ablated in a single exposure, since it works by focusing high-energy US waves on a volume of tissue approximately the size of a grain of rice. When larger amounts of tissue are to be ablated, as in tumor therapy, it results in long treatment periods and thereby adversely affects the patient's quality of life. Furthermore, the clinical applications of HIFU are limited due to the rarity of an adequate acoustic window to access the tumors, or inevitable injuries to the adjacent structures.

The microbubbles (Mbs) contain gas encased in a shell, and have diameters between approximately 1 and $5 \mu \mathrm{m}$ so that they are capable of passing through the capillary network. When the bubbles pass through the tissue volume and are exposed to US, they expand and contract at the frequency of the propagating acoustic wave due to the cyclic pressure reductions and increases associated with the wave propagation. The bubble's oscillation also causes the surrounding fluid to flow (microstreaming), thus creating large shear forces around the bubbles. In addition, the bubbles are pushed by a radiation force in the direction of wave propagation $(7,8)$. Above a particular threshold, the bubble's oscillation becomes so intense that the inertia of the surrounding fluid causes the bubble to collapse, inducing high temperatures and pressures. The result is a shock wave, which propagates at supersonic speed from the collapse 
site. If the bubbles collapse near a vessel wall, they may create fluid jets, which are likely to puncture the wall (9-11). As a result, the bubbles absorb and concentrate energy from the US wave into a microscopic tissue volume, reducing the US power levels by at least two orders of magnitude from that required to induce bio-effects without the bubbles (12).

The purpose of this study was to evaluate the anti-tumor effect of low frequency and low intensity US radiation combined with $\mathrm{Mb}$ intravenous injection on prostate tumors subcutaneously implanted in nude mice.

\section{Materials and methods}

Experimental animals. A total of 40 male Balb-c nude mice (5 weeks old; weight 18-25 g) were obtained from the Experimental Animal Center of Shanghai (Shanghai, China). Animal experiments were approved by the Ethics Committee of Laboratory Animal Welfare of Shanghai Jiaotong University, Shanghai, China.

Animal model. The Du145 cell line was obtained from the cell library of the Chinese Academy of Sciences (Shanghai, China). A total of $2 \times 10^{7}$ Du145 cells in $200 \mu$ l of phosphate-buffered saline were injected subcutaneously into the right flank region of the nude mouse to establish a tumor model. When the tumors grew to approximately $10 \mathrm{~mm}$ in maximum diameter, 40 mice were randomly divided into 4 groups of 10 each: the US+Mbs group, US group, Mbs group and control group.

Microbubbles. SonoVue ${ }^{\circledR}$ (Bracco Company, Italy) was used. After the plastic cap of the vial was removed, $5 \mathrm{ml}$ sterile normal saline was added into the vial. The vial was agitated vigorously for approximately $20 \mathrm{sec}$ before the milky white suspension was ready.

US equipment and experimental procedures. The Ultrasonics Processing FS4500 US Tumor Therapeutic System (Fudan Institute Technology, China) was used in this study. The parameters used were: US frequency $20 \mathrm{kHz}$, ISP $200 \mathrm{~mW} / \mathrm{cm}^{2}$. In the US+Mbs group, $0.2 \mathrm{ml}$ SonoVue was injected slowly via tail veins, followed by rapid injection of $0.2 \mathrm{ml}$ normal saline as the power generator was turned on; the whole target tumor was irradiated for $120 \mathrm{sec}$. In the US group, the same US was applied to tumors as the US+Mbs group, but it was combined with intravenous injection of $0.4 \mathrm{ml}$ normal saline only. In the Mbs group, the mice were intravenously injected with $0.2 \mathrm{ml}$ SonoVue and $0.2 \mathrm{ml}$ normal saline, but without US exposure. No interventions were performed on the control group. The treatments were repeated three times in total every other day.

Tumor challenge. From the first day of treatment, the tumor growth was monitored, and its diameter was measured every 3rd day by a US machine (ESAOTE MyLab 90, Genoa, Italy). The tumor volume was then calculated using the formula: tumor volume $\left(\mathrm{mm}^{3}\right)=\mathrm{d}^{2} \mathrm{xD} / 2$, where $\mathrm{d}$ and $\mathrm{D}$ are the shortest and longest diameters of the measured tumor, respectively. Tumor inhibition ratio was calculated using the formula: tumor volume inhibition ratio $(\%)=\left(\mathrm{V}_{1}-\mathrm{V}_{2}\right) / \mathrm{V}_{2}$, where $\mathrm{V}_{1}$ and $\mathrm{V}_{2}$ are the tumor volume of therapy groups and average volume of control group, respectively. Differences were tested with analysis of variance or the Student's t-test, and results were considered to be statistically significant at $\mathrm{P}<0.05$.

Specimens. Two weeks following the intial treatment, all of the mice in the four groups were sacrificed and tumors were surgically excised. Sections $\left(1 \mathrm{~mm}^{3}\right)$ were sampled, double fixed by glutaraldehyde and osmium acid, embedded in epoxy resin and sectioned ultra-thinly. Ultra-structural changes of the targeted tissues were observed under a transmission electron microscope. The remaining tumor tissues were stained with hematoxylin and eosin (H\&E) and observed under a light microscope.

Immunohistochemical examination. H\&E staining and immunohistochemical examination were performed. The microvessel density (MVD) of the tumor was calculated under microscopy by marking the tumor vessels with mouse anti-human CD34 monoclonal antibody according to Weidner's revised technique (13). The expression of vascular endothelial growth factor (VEGF) was marked with the mouse anti-human VEGF monoclonal antibody. The whole slide was viewed at 100-times field of view (FOV) under the microscope, and the 'hot-spot' (i.e., the most intensive area of tumor angiogenesis) was found. Then, under 200-times FOV, the number of tumor vessels was calculated and averaged as MVD by viewing 5 FOV randomly on each slide. The same method was used to calculate the average optical density (AOD) of VEGF. The observed data were exhibited as the mean \pm standard deviation. Statistical analysis was performed using a SPSS 13.0 statistical software package. The difference of MVD counts and AOD of VEGF in the tumor tissue of 40 mice in 4 groups were obtained using the one-way analysis of variance. Differences were considered to be statistically significant at $\mathrm{P}<0.05$.

\section{Results}

Gross observation. Each tumor was detected with US when the tumor reached a diameter of approximately $0.5 \mathrm{~cm}$ in muscle, 14 days after the Du145 prostate tumor cells were injected subcutaneously. A total of 28 days after the tumor cells were implanted, the diameter of the tumors ranged from 7 to $11 \mathrm{~mm}$. The implanted tumors were observed to be spherical-, elliptical- or nodular-shaped by US sonography.

In the control group, the average tumor volume markedly increased at the time of 2 weeks compared to the US+Mbs group $(\mathrm{P}<0.05)$. The average tumor volume inhibition ratio of the US+Mbs group was $62.70 \%$, which was significantly greater than that of the Mbs group (16.34\%) and the US group $(23.66 \%)$. However, the average tumor volume of the US group and the Mbs group at 2 weeks was similar to the control group $(\mathrm{P}>0.05)$.

Gross pathological findings and light microscopy. In the US, Mbs and control groups, the tumors resembled the appearance of gray fish meat and the cells were encapsulated. A clear demarcation with a sharp boundary was detected between the tumor and normal surrounding tissues. In the US+Mbs group, there was a large amount of yellowish coagulation necrosis inside the tumor. 
A

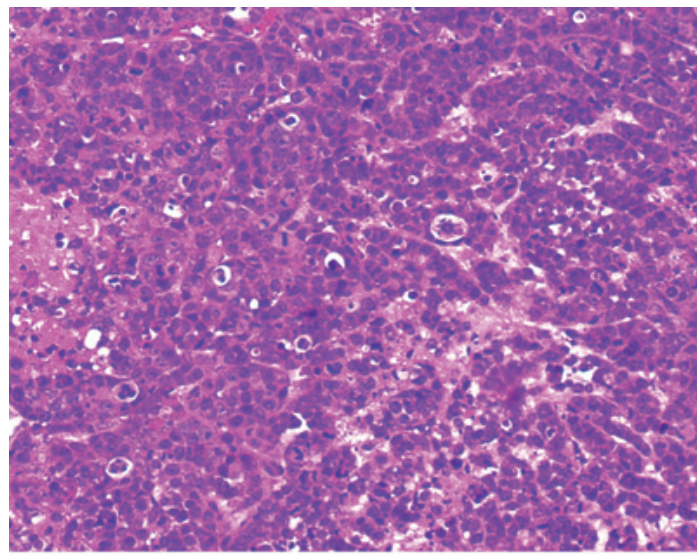

C

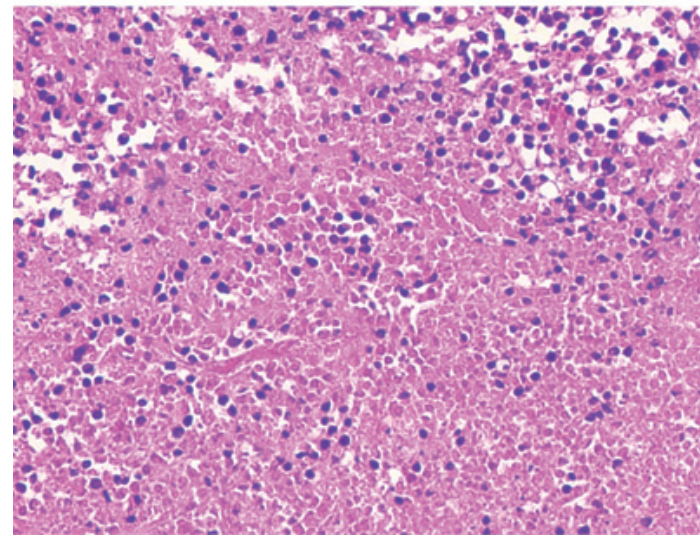

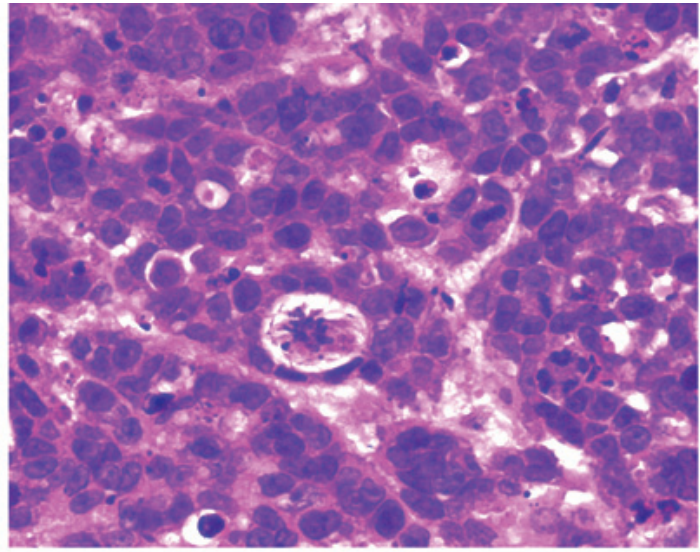

D

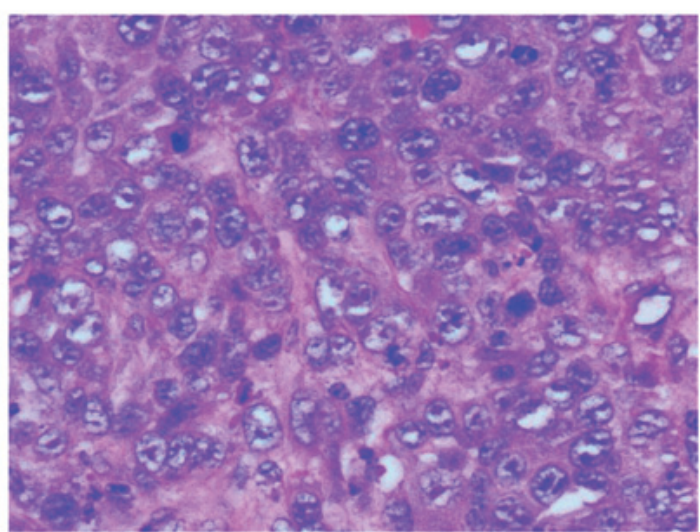

Figure 1. Light microscopic pathology of the targeted prostate tumor tissues of node mice in the control and US+Mbs groups (H\&E, A and C; magnification, x100; B and D; magnification, x400). (A and B) In the control group, tumor cells were large, irregularly arranged and with irregular morphology. The nuclei were large and deeply H\&E-stained, with a large karyoplasmic ratio and increased mitosis. (C and D) In the US+Mbs group, the tumor cytoplasm in all 10 mice was lightly stained, with cytoplasmic vacuoles of various sizes, chromatin margination and karyopyknosis. US, ultrasound; Mbs, microbubbles; H\&E, hematoxylin and eosin.

On the H\&E-stained slides, under a low power lens, Du145 prostate tumor cells in the control group appeared mass-flake-like or as an invasive cancer nest, with reduced connective tissues and an unclear demarcation between the tumor and mesenchymal cells. Under a high-power lens, tumor cells were large, irregularly arranged with an irregular morphology. The nuclei were large and deeply stained, with a large karyoplasmic ratio and increased mitosis. The tumor tissue of the US and Mbs groups was similar to that of the control group. The tumor cells of the US+Mbs group were shrunk by coagulation necrosis, and their volume was reduced. Some residual tumor cells remained in the periphery of the tumors. The tumor cytoplasm was lightly stained with cytoplasmic vacuoles of various sizes (Fig. 1).

Electron microscopy. In the control group, a high magnification view of the wall of a tumor blood vessel in the control group showed highly attenuated vascular endothelium. The nuclei of the endothelial cells were large and deformed, with clear nuclear membranes and rich euchromatin. The chromatin particles were large with intranuclear pseudo-inclusions and multiple visible nucleoli were present. No difference was observed in the appearance of the US and Mbs groups compared with the control group. In the US+Mbs group, changes in the wall perimeter included small membrane blebs, unusual vacuoles or multiple filopodia, small gaps in the endo- thelial layer and regions of disrupted or missing endothelium. The diameter of these gaps ranged from hundreds of nanometers to several microns. Endothelial cells were reduced in size, and in certain endothelial cells, karyopyknosis was revealed and various vacuoles of different sizes were present in the cytoplasm (Fig. 2).

Immunohistochemical examination. CD34 expression was located on the vascular endothelial cells of the tumor. By targeting the microvessels with CD34 monoclonal antibody, a large amount of tumor microvessel density was exhibited as a brown-yellow dying area on the immunohistochemical slides in the control group. In the US+MBs group, the microvessels of the tumor were dispersed and exiguous, and the MVD markedly decreased compared with that of the control group.

The expression of VEGF was mainly located in the plasma of the tumor cells. A positive expression of VEGF appeared as brown-yellow slender particles. A decreased expression level of VEGF in tumor cells was observed in the US+Mbs group compared to the control group. A small amount of the brownyellow granular substance was detected in part of the cytoplasm.

As shown in Table I, the amount of CD34- and VEGFpositive expression in US+Mbs significantly decreased when compared with that of the control, US and MBs groups $(\mathrm{P}<0.05)$, suggesting that $\mathrm{US}+\mathrm{Mbs}$ was capable of inducing the inhibition of angiogenesis. However, no differences were 

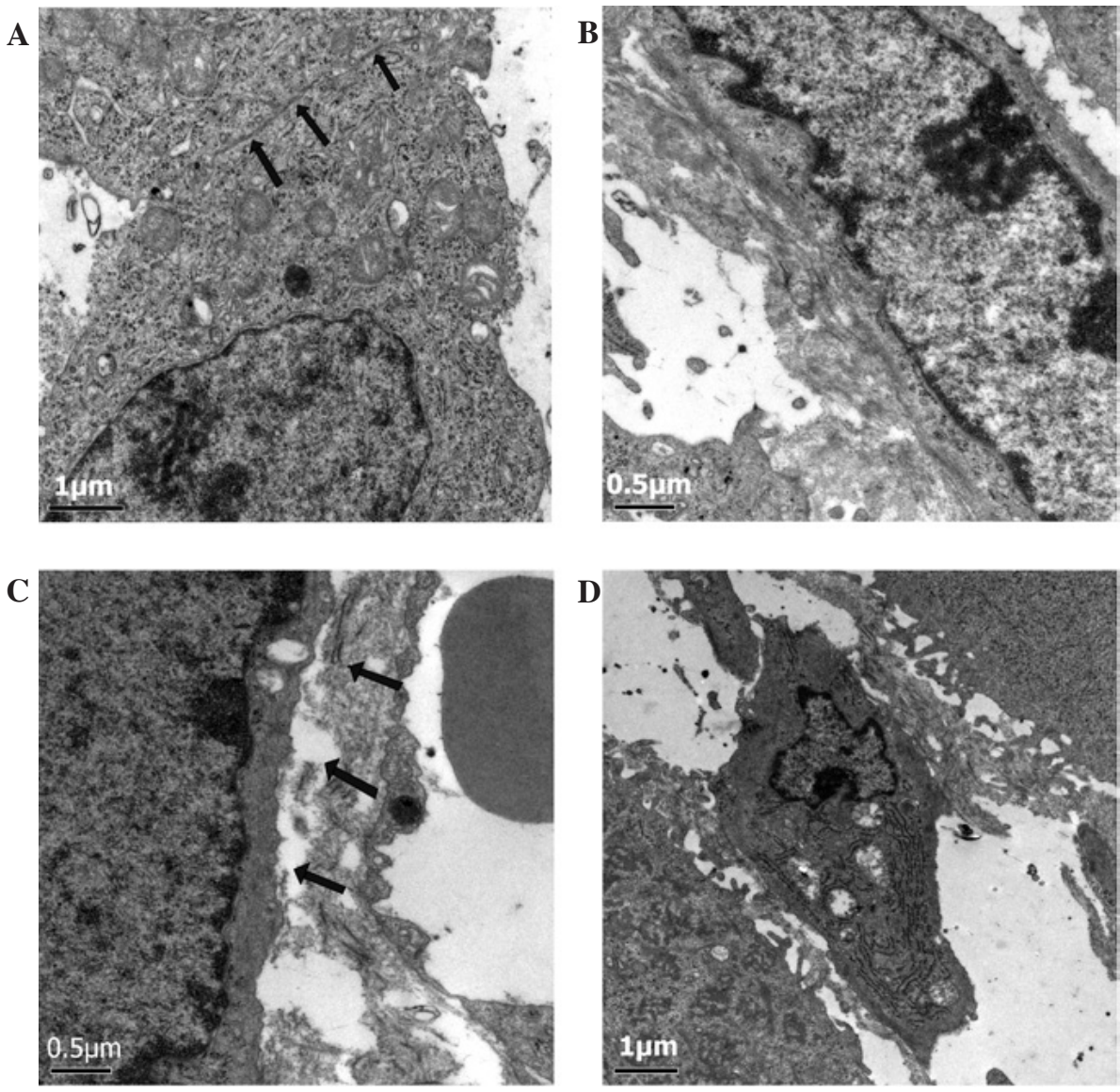

Figure 2. Transmission electron micrographs show the range of effects of US+Mbs treatment in a mouse model. (A) Image of the control group shows tight junctions of vascular endothelium (arrows). (B) High magnification view of the vessel basement membrane in the control group. (C) Image of the US+Mbs group shows a gap in the endothelial layer and separation from the underlying intact pericyte (arrows). (D) View of vacuoles and multiple filopodia of an endothelium cell in the US+Mbs group. US, ultrasound; Mbs, microbubbles.

Table I. The tumor vessel counts of CD34- and VEGF-positive expression in the different groups.

\begin{tabular}{lcc}
\hline Group & MVD of CD34 & AOD of VEGF \\
\hline Control & $32.50 \pm 3.05$ & $29.34 \pm 7.70$ \\
US & $26.53 \pm 6.58^{\mathrm{d}}$ & $28.4 \pm 6.77^{\mathrm{d}}$ \\
Mbs & $26.73 \pm 2.37^{\mathrm{d}}$ & $28.18 \pm 5.68^{\mathrm{d}}$ \\
US+Mbs & $3.30 \pm 1.84^{\mathrm{a}, \mathrm{b}, \mathrm{c}}$ & $5.35 \pm 2.85^{\mathrm{a}, \mathrm{b}, \mathrm{c}}$ \\
\hline
\end{tabular}

${ }^{\mathrm{a}} \mathrm{P}<0.05$ vs. control, ${ }^{\mathrm{b}} \mathrm{P}<0.05$ vs. Mbs group, ${ }^{\mathrm{c}} \mathrm{P}<0.05$ vs. US group, ${ }^{\mathrm{d}} \mathrm{P}>0.05$ vs. control. MVD, microvessel density; AOD, average optical density; US, ultrasound; Mbs, microbubbles.

observed in the expression of CD34 and VEGF between the US, Mbs and control groups, respectively ( $\mathrm{P}>0.05)$.

\section{Discussion}

Physical therapy applications using Mbs and US cavitation on the disruption of tumor neovasculature have drawn much attention due to their use in gene transfection, targeted drug delivery and release, and thrombolysis (14-20). The ultrasonic cavitation effect is a significant physical effect of US, besides the thermal effect. Mbs as an effective cavitation core may induce a significant cavitation effect under appropriate ultrasonic impulse excitation. Cavitation-released mechanical energy (non-thermal effect) has the potential of ablating targeted tissue. This hypothesis suggests disrupting the immature, leaky and fragile tumor microvasculature is possible. In addition, being a simple physical therapeutic method, Mbs enhanced US cavitation to obstruct tumor microcirculation can be repeated with equal success and may be capable of preventing the thermal side effects of HIFU treatment.

In our study, the prostate tumors of nude mice treated with low-intensity US combined with the intravenous injection of Mbs were ablated by non-thermal effects, which have characteristic pathological changes that are different from those of thermal lesions. Ashush et al (21) observed the following morphological changes after US cavitation: cell shrinkage, vacuole formation, chromatin condensation, karyorrhexis and the formation of apoptotic bodies. Kieran et al (22) studied non-thermal lesions by changing the US intensity and duty cycle. Their histological observation showed that within a certain intensity and duty cycle, vacuoles were formed in the cells, with blanched and dense liquid inside the vacuoles. Our study found that neither low intensity US nor Mbs, as separate conditions, were able to achieve a tumor ablation effect. However, when the two factors were combined together, the tumor inhibition effect was significant. Light microscopy 
showed abundant vacuoles of various sizes in the cytoplasm and chromatin margination and karyopyknosis in certain cells. Electron microscopic examination revealed a presence of karyopyknosis and chromatin margination in certain cells, intercellular space widening, and a number of vacuoles of various sizes in the cytoplasm. These findings indicated that by combining low frequency US with Mbs, cavitation effects may be intensified to achieve non-thermal tumor ablation.

VEGF is known to be a potent stimulator of endothelial cell proliferation, vascular permeability and angiogenesis. VEGF may be stimulated by the platelet-derived growth factor and function synergistically with the fibroblast growth factor to stimulate new vessel growth. Inhibition of the VEGF receptor tyrosine kinase activity has been shown to slow the tumor growth in various tumor models, including metastatic colon cancer, mammary and pancreatic adenocarcinomas (23-27). It is likely that by targeting and disrupting the receptor tyrosine kinase activity of multiple angiogenic modulators, such as VEGF, platelet-derived growth factor and fibroblast growth factor, may more effectively inhibit tumor growth. A distinct increase in the expression levels of promoting factors of angiogenesis, such as VEGF, has been observed during tumor growth and evolution. VEGF is capable of specially binding the corresponding acceptor of vascular endothelial cells and promoting the proliferation of vascular endothelial cells. Moreover, it increases the permeability of vessels and facilitates the exudation of serous protein including fibrinogen (28). Accordingly, during contrast-enhanced low frequency and low intensity US therapy, US cavitation inhibited the expression of VEGF in prostate tumors in nude mice.

Contrast-enhanced low frequency and low intensity US cavitation produced injury of vascular endothelial cells in prostate tumors, and inhibited the expression of VEGF in the tumor, resulting in tumor inhibition effects. The potential for such effects during contrast-enhanced US cavitation at $20 \mathrm{kHz}$ should be acknowledged. The major application of this study is in the target therapy of solid tumors with abundant microvessels. Future studies are required into certain aspects of US cavitation, such as cavitation detection, temperature monitoring and other means to detect non-thermal effects; how to optimize the combination between US and Mbs exposure parameters; the means to control and monitor cavitational lesions; and longterm outcomes of non-thermal tumor ablation.

\section{Acknowledgements}

This study was supported by the Natural Science Foundation of Shanghai (grant 10JC14125600).

\section{References}

1. Lynn JG, Zwemer RL, Chick AJ and Miller AE: A new method for the generation and use of the focused ultrasound in experimental biology. J Gen Physiol 26: 179-193, 1942.

2. Valtot F, Kopel J and Haut J: Treatment of glaucoma with high-intensity focused ultrasound. Int Ophthalmol 13: 167-170, 1989.
3. Kincaid LF, Sanghvi NT, Cummings O, et al: Noninvasive ultrasound subtotal ablation of the prostate in dogs. Am J Vet Res 57: 1225-1227, 1996

4. Bihrle R, Foster RS, Sanghvi NT, Fry FJ and Donohue JP: High-intensity focused ultrasound in the treatment of prostatic tissue. Urology 43: 21-26, 1994.

5. Madersbacher S, Pedevilla M, Vingers L, Susani M and Marberger M: Effect of high-intensity focused ultrasound on human prostate cancer in vivo. Cancer Res 55: 3346-3351, 1995.

6. Vallancien G, Chartier-Kastler E, Harouni M, Chopin D and Bougaran J: Focused extracorporeal pyrotherapy: experimental study of feasibility in man. Semin Urol 11: 7-9, 1993.

7. Hynynen K, McDannold N, Vykhodtseva N, et al: Focal disruption of the blood-brain barrier due to $260-\mathrm{kHz}$ ultrasound bursts: a method for molecular imaging and targeted drug delivery. J Neurosurg 105: 445-454, 2006.

8. Dayton P, Klibanov A, Brandenburger G and Ferrara K: Acoustic radiation force in vivo: a mechanism to assist targeting of microbubbles. Ultrasound Med Biol 25: 1195-1201, 1999.

9. Apfel RE: Acoustic cavitation: A possible consequence of biomedical use of ultrasound. Br J Cancer Suppl 5: 140-146, 1982.

10. Crum LA and Fowlkes JB: Acoustic cavitation generated by microsecond pulses of ultrasound. Nature 319: 52-54, 1986.

11. Margulis MA: Sonochemistry of Cavitation. Gordon and Breach Publishers, Luxembourg, 1995.

12. Hynynen K, McDannold N, Vykhodtseva N and Jolesz FA: Noninvasive MR imaging-guided focal opening of the bloodbrain barrier in rabbits. Radiology 220: 640-646, 2001.

13. Weidner N: Tumor vascularity and proliferation: clear evidence of a close relationship. J Pathol 189: 297-299, 1999.

14. Mayer S and Grayburn PA: Myocardial contrast agents: recent advances and future directions. Prog Cardiovasc Dis 44: 33-44, 2001.

15. Unger EC, Hersh E, Vannan M, Matsunaga TO and McCreery T: Local drug and gene delivery through microbubbles. Prog Cardiovasc Dis 44: 45-54, 2001.

16. Porter TR and Xie F: Therapeutic ultrasound for gene delivery. Echocardiography 18: 349-353, 2001.

17. Lindner JR and Kaul S: Delivery of drugs with ultrasound. Echocardiography 18: 329-337, 2001.

18. Tachibana K and Tachibana S: The use of ultrasound for drug delivery. Echocardiography 18: 323-328, 2001.

19. Unger EC, Matsunaga TO, McCreery T, Schumann P, Sweitzer R and Quigley R: Therapeutic applications of microbubbles. Eur J Radiol 42: 160-168, 2002.

20. Price RJ and Kaul S: Contrast ultrasound targeted drug and gene delivery: an update on a new therapeutic modality. J Cardiovasc Pharmacol Ther 7: 171-180, 2002.

21. Ashush H, Rozenszajn LA, Blass M, et al: Apoptosis induction of human myeloid leukemic cells by ultrasound exposure. Cancer Res 60: 1014-1020, 2000.

22. Kieran K, Hall TL, Parsons JE, et al: Refining histotripsy: defining the parameter space for the creation of nonthermal lesions with high intensity, pulsed focused ultrasound of the in vitro kidney. J Urol 178: 672-676, 2007.

23. Hicklin DJ and Ellis LM: Role of the vascular endothelial growth factor pathway in tumor growth and angiogenesis. J Clin Oncol 23: 1011-1027, 2005.

24. Fidler IJ and Ellis LM: The implications of angiogenesis for the biology and therapy of cancer metastasis. Cell 79: 185-188, 1994.

25. Bergers $\mathrm{G}$ and Benjamin LE: Tumorigenesis and the angiogenic switch. Nat Bev Cancer 3: 401-410, 2003.

26. Hanahan D and Weinberg RA: The hallmark of cancer. Cell 100: $57-70,2000$.

27. Nyberg P, Xie L and Kalluri R: Endogenous inhibitors of angiogenesis. Cancer Res 65: 3967-3979, 2005.

28. Ferrara N, Gerber HP and LeCouter J: The biology of VEGF and its receptors. Nat Med 9: 669-676, 2003. 